\title{
Regularized multiframe phase-shifting algorithm for three-dimensional profilometry
}

\author{
Fuqin Deng, ${ }^{1,2}$ Wui Fung Sze,${ }^{2}$ Jiangwen Deng, ${ }^{2}$ Kenneth S. M. Fung, ${ }^{2}$ \\ W. H. Leung, ${ }^{2}$ and Edmund Y. Lam ${ }^{1, *}$ \\ ${ }^{1}$ Imaging Systems Laboratory, Department of Electrical and Electronic Engineering, \\ University of Hong Kong, Pokfulam Road, Hong Kong 999077, China \\ ${ }^{2}$ ASM Assembly Automation Ltd., Kwai Chung, Hong Kong 999077, China \\ *Corresponding author: elam@eee.hku.hk
}

Received 27 June 2011; revised 25 August 2011; accepted 26 August 2011;

posted 2 September 2011 (Doc. ID 149956); published 22 December 2011

\begin{abstract}
In many industrial inspection systems, it is required to have a high-precision three-dimensional measurement of an object under test. A popular technique is phase-measuring profilometry. In this paper, we develop some phase-shifting algorithms (PSAs). We propose a novel smoothness constraint in a regularization framework; we call this the R-PSA method and show how to obtain the desired phase measure with an iterative procedure. Both the simulation and experimental results verify the efficacy of our algorithm compared with current multiframe PSAs for interferometric measurements. (c) 2011 Optical Society of America
\end{abstract}

OCIS codes: $110.6880,150.3040,120.6660$.

\section{Introduction}

Measuring the surface profile of an object is a very useful procedure in inspection systems as it often sheds light on whether there exists defects or misalignments or even potential connection problems in the forthcoming manufacturing steps. This is particularly challenging when applied to integrated circuit (IC) packages due to two conflicting requirements: a small feature size requiring high precision and the need to a high throughput [1]. There are several general approaches to optical noncontact three-dimensional reconstruction, namely shape from shading (SFS), confocal imaging, stereo, and structured light reconstruction. Not all of them are appropriate when we restrict our attention on reconstructing the surfaces only. The SFS technique involves heavy computations and is intrinsically ill posed, and it has been argued that most of the assumptions are inappropriate for reconstructing surfaces [2]. Confocal imaging can statically reconstruct

0003-6935/12/010033-10\$15.00/0

(C) 2012 Optical Society of America an object profile with high accuracy without occlusion [3] but is not suitable for high-speed measurement due to its sequential acquisition nature. Area-based stereo allows us to obtain a dense surface map, but multiple cameras are needed, and this approach fails at occluded regions or within featureless regions [4].

So far, methods based on structured light and combined with a triangular setup are the most appropriate for surface profilometry. Specially designed patterns are projected on the object; these patterns can be coded in many ways for different considerations such as resolution, speed, and robustness. For instance, laser triangulation projects a dot or a line on the surface of the object. To get an accurate surface profile, a high-resolution laser light source and a high-resolution camera are both needed. However, this method is slow since it involves scanning the whole surface point by point or line by line. An improvement can be made if we project grayscale stripes, color stripes, or dot matrix patterns, where the more sophisticated approaches mean we can reduce the number of images needed to reconstruct the 
three-dimensional profile [5]. In fact, projecting a sinusoidal pattern is perhaps the most common scheme, where efficient reconstruction methods exist, namely Fourier transform profilometry and phase-measuring profilometry (PMP) based on Fourier transform and analysis [6]. The latter has attracted much interest particularly because it can deliver a high-precision reconstruction [7].

Meanwhile, projecting binary stripes temporally or spatially represents another interesting advance in optical projection technology $[8,9]$. These binary patterns allow the reconstruction to be insensitive to quantization error and noise from the camera. For example, using a Hamming error-correcting code, we can perform robust surface reconstruction with some error correction capability [10]. The reconstruction needs an additional interpolation step to obtain a smooth surface of the object due to the discrete nature of the patterns. For us to obtain sufficient sample points for better interpolation, we either need to acquire more images or use higher-resolution patterns. The second option is sometimes not realistic because high-resolution binary patterns are blurred after passing through a diffraction-limited optical system, and they also bring additional difficulties in establishing the correspondence in the decoding stage.

In this paper, we focus on techniques that use structured light for three-dimensional reconstruction and particularly on the phase-shifting algorithms (PSAs) in PMP. Our primary application is on semiconductor die inspection, although the scheme can be applied to other application areas as well. In certain inspections, such as measuring the height of wafer bumps, explicit three-dimensional reconstruction is not needed, and various efficient methods have been proposed. For example, a biplanar disparity matrix measure was developed to compute the height based on a specially designed lighting setup [11]. Also, binary patterns were projected on the surface of the IC samples in order to obtain more sampled profile data [12]. However, if a more complete profile information is needed, such as in surface inspection and volume measurement, we need ways to reconstruct the full height profile.

\section{Model for PMP}

Figure 1 shows the setup of the three-dimensional phase reconstruction system. Light is projected through the sinusoidal grating, reflected on the object surface, and received by the camera. If the object were absent, the camera would have captured light emanating from point $C$ on the reference plane, but in this case, the light reflected from point $D$ is captured instead. Accordingly, the phase of the sinusoidal pattern received at the sensor varies with the height of the surface. With the triangular optical setup, when the projecting and imaging systems are both telecentric, the relationship between the surface height $h(x, y)$ (where $x$ and $y$ denote the coordinates on the base plane of the object) and the corresponding phase offset $\phi_{h}(x, y)$ can be modeled as

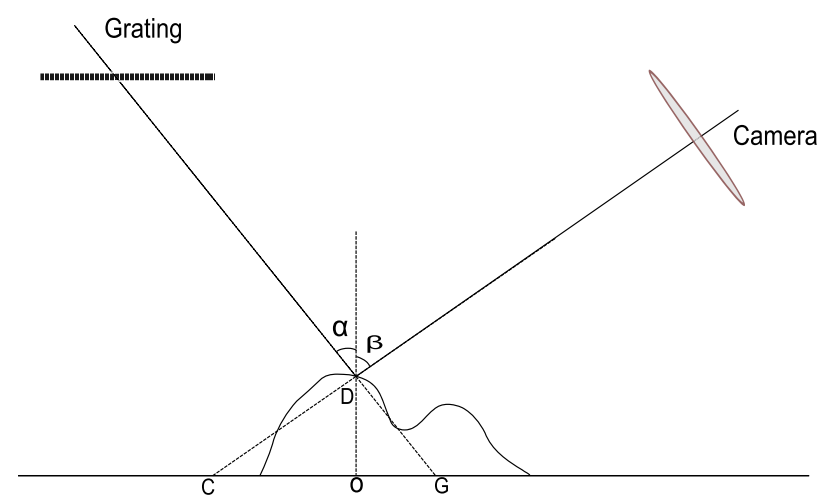

Fig. 1. (Color online) Setup for the three-dimensional phase reconstruction system.

$$
\phi_{h}(x, y)=\frac{2 \pi(\tan \alpha+\tan \beta)}{P} h(x, y),
$$

where $\alpha$ and $\beta$ are the incident angles of the projector and the camera, respectively, and $P$ is the pitch of the grating on the reference surface [13].

This phase offset, when added to a reference surface phase $\phi_{r}(x, y)$, becomes the total phase of the sinusoidal signal, denoted $\phi(x, y)$. Thus, we have

$$
\phi(x, y)=\phi_{r}(x, y)+\phi_{h}(x, y) .
$$

Now if we assume $B(x, y)$ is the background intensity and $F(x, y)$ is the fringe contrast, the captured image $I_{1}(x, y)$ is given by

$$
I_{1}(x, y)=B(x, y)+F(x, y) \cos \phi(x, y)+N_{1}(x, y),
$$

where $N_{1}(x, y)$ is additive noise. The subscript " 1 " is there because this is the first image we would obtain. In this equation, we have three variables, namely $B(x, y), F(x, y)$, and $\phi(x, y)$. Therefore, we need at least three images to solve for them. In practice, it is common to use four, with phase shifts at $\pi / 2$ apart. We therefore have the following four acquired images, i.e.,

$$
\begin{aligned}
I_{k}(x, y)= & B(x, y)+F(x, y) \cos [\phi(x, y) \\
& +(k-1) \pi / 2]+N_{k}(x, y),
\end{aligned}
$$

where $k=\{1,2,3,4\}$.

In the absence of noise, the above equations imply that

$$
\tan \phi(x, y)=\frac{I_{4}(x, y)-I_{2}(x, y)}{I_{1}(x, y)-I_{3}(x, y)}
$$

and therefore we have

$$
\phi_{h}(x, y)=\arctan \left\{\frac{I_{4}(x, y)-I_{2}(x, y)}{I_{1}(x, y)-I_{3}(x, y)}\right\}-\phi_{r}(x, y) .
$$

The surface height is then recovered with Eq. (1). 
This analytical solution allows for high-speed phase reconstruction. However, in reality, errors due to spurious reflection, intensity fluctuations, phaseshift error, system vibration, camera noise, quantization error, and image blurring often cause this algorithm to fail. Many extensions of the above formulation have been made over the years. For example, Ai and Wyant investigated the effect of spurious reflection on phase-shifting interferometry [14], while Brophy considered the effect of the intensity fluctuations on the PSA based on Taylor expansion [15]. Subsequently, de Groot analyzed the effect of the mechanical vibrations and phase-shift error based on Fourier analysis [16]. Meanwhile, to improve the accuracy and the robustness of the fourframe algorithm, researchers have proposed various extensions, such as five-frame algorithms [17]. With more images, they can further explore the statistical properties and the interframe correlation of the images. However, acquiring more images slows down the whole system and complicates the optical design.

In what follows, we argue that the fringe contrast $F(x, y)$ is a key factor in accurate phase reconstruction. Since it is coupled with the phase as in Eq. (3), when the fringe contrast is small, the reconstructed phase will be unstable and inaccurate. For instance, in the low signal-to-noise ratio (SNR) regions such as the dark background regions, which can include the substrate regions in our IC packaging application and the occluded regions, the fringe contrast is small due to low reflectivity. The situation is more serious after blurring because the fringe contrast will be reduced further. However, surface reconstruction at the low fringe contrast region is often important such as for surface defect inspection and volume measurement. Therefore, a tilted optics system has been designed to improve the image quality [18]. In this paper, we improve the accuracy and robustness for the surface profile from a signal processing point of view by solving a constrained optimization problem.

\section{Regularized Multiframe PSA}

\section{A. Optimization Formulation}

Let us generalize the derivation above and consider an $n$-frame PSA, where each frame $I_{k}(x, y)$ is captured with a shift $s_{k}$ (for $\left.k=1, \ldots, n\right)$. The four-frame algorithm earlier would correspond to $n=4$ and $s_{k}=(k-1) \pi / 2$. Let $E_{k}(x, y)$ be the residual error of the model, i.e.,

$$
\begin{aligned}
E_{k}(x, y)= & I_{k}(x, y)-\{B(x, y) \\
& \left.+F(x, y) \cos \left[\phi(x, y)+s_{k}\right]\right\} .
\end{aligned}
$$

We aim to find $B(x, y), F(x, y)$, and $\phi(x, y)$ for each pixel location $(x, y)$ in the following optimization framework by minimizing

$$
E(x, y)=\sum_{k=1}^{n} E_{k}^{2}(x, y) .
$$

However, due to nonlinearity, it is difficult to obtain the global optimal solution $\phi(x, y)$, and we may be trapped in local optimal solutions instead.

\section{B. Change of Variables}

We let $F_{c}(x, y)=F(x, y) \cos \phi(x, y)$ and $F_{s}(x, y)=$ $F(x, y) \sin \phi(x, y)$. As such, if we consider $B(x, y)$, $F_{c}(x, y)$, and $F_{s}(x, y)$ as variables, the cost function in Eq. ( ) is quadratic, and we can obtain the global optimization using convex optimization [19]. However, this change of variables comes with some cost: there must be an additional constraint to impose on the variables.

We rewrite Eq. (7) by expanding on the trionometric function as

$$
\begin{aligned}
E_{k}(x, y)= & I_{k}(x, y)-\left\{B(x, y)+F_{c}(x, y) \cdot\left(\cos s_{k}\right)\right. \\
& \left.+F_{s}(x, y) \cdot\left(-\sin s_{k}\right)\right\} .
\end{aligned}
$$

Consider a specific point $\left(x_{0}, y_{0}\right)$. If we let

$$
\begin{aligned}
& \mathrm{M}_{0}= {\left[\begin{array}{ccc}
1 & \cos s_{1} & -\sin s_{1} \\
1 & \cos s_{2} & -\sin s_{2} \\
\vdots & \vdots & \vdots \\
1 & \cos s_{n} & -\sin s_{n}
\end{array}\right], } \\
& \mathbf{d}_{0}=\left[\begin{array}{c}
I_{1}\left(x_{0}, y_{0}\right) \\
I_{2}\left(x_{0}, y_{0}\right) \\
\vdots \\
I_{n}\left(x_{0}, y_{0}\right)
\end{array}\right], \text { and } \mathbf{v}_{0}=\left[\begin{array}{c}
B\left(x_{0}, y_{0}\right) \\
F_{c}\left(x_{0}, y_{0}\right) \\
F_{s}\left(x_{0}, y_{0}\right)
\end{array}\right],
\end{aligned}
$$

then solving for the minimum of

$$
E\left(x_{0}, y_{0}\right)=\left\|\mathrm{M}_{0} \mathbf{v}_{0}-\mathbf{d}_{0}\right\|_{2}^{2}
$$

is equivalent to the optimization problem presented in Eq. (8). Note that the matrix $\mathrm{M}_{0}$ does not depend on the pixel location, and therefore it can be stored once for a specific set of translations $\left\{s_{k}\right\}$.

\section{Four-Frame Example}

To elucidate the methodology further, let us consider the four-frame capture with $s_{k}=(k-1) \pi / 2$. The matrix $M_{0}$ is then

$$
\mathrm{M}_{0}=\left[\begin{array}{ccc}
1 & \cos 0 & -\sin 0 \\
1 & \cos \frac{\pi}{2} & -\sin \frac{\pi}{2} \\
1 & \cos \pi & -\sin \pi \\
1 & \cos \frac{3 \pi}{2} & -\sin \frac{3 \pi}{2}
\end{array}\right]=\left[\begin{array}{ccc}
1 & 1 & 0 \\
1 & 0 & -1 \\
1 & -1 & 0 \\
1 & 0 & 1
\end{array}\right]
$$

Furthermore, if we treat Eq. (11) as an unconstrained optimization problem, we can in fact compute the solution via the analytical formula 


$$
\mathbf{v}_{0}=\left(\mathrm{M}_{0}^{T} \mathrm{M}_{0}\right)^{-1} \mathrm{M}_{0}^{T} \mathbf{d}_{0}
$$

which yields

$$
\begin{aligned}
B\left(x_{0}, y_{0}\right)= & \frac{1}{4}\left[I_{1}\left(x_{0}, y_{0}\right)+I_{2}\left(x_{0}, y_{0}\right)+I_{3}\left(x_{0}, y_{0}\right)\right. \\
& \left.+I_{4}\left(x_{0}, y_{0}\right)\right]
\end{aligned}
$$

We can then recover the phase $\phi\left(x_{0}, y_{0}\right)$ in the range $[-\pi, \pi)$ from $\cos \phi\left(x_{0}, y_{0}\right)$ and $\sin \phi\left(x_{0}, y_{0}\right)$.

\section{Analyses and Refinements}

It may not seem to be much of a difference if we compare Eqs. (15) and (16) with Eq. (5); after all, both expressions are derived for a noiseless case, and the former implies the latter. However, there are actually several advantages to use the optimization formulation.

- It allows for a general shifting strategy. In a real system, the captured images may not be located at the designed shift values due to motion error, and some real-time processes, such as defect analysis, may cause the fringe images to be captured at uneven shift values. A situation such as $s_{1}=0$, $s_{2}=\pi / 8, s_{3}=13 \pi / 8, s_{4}=15 \pi / 8$ would not be atypical. In the optimization setting, we can still obtain the global optimal solution at these phase shifts.

- With a matrix formulation, we can analyze the numerical stability resulting from various phase shifts. For example, the even shift in the earlier example gives a $M_{0}$ with a condition number of

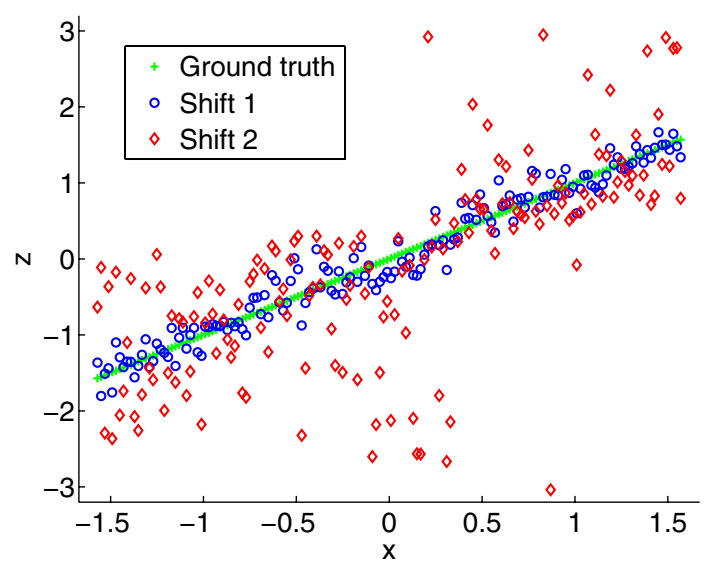

Fig. 2. (Color online) Reconstruction results measured in radians from two sets of four-frame phase shifts. "Shift 1" is $\{0, \pi / 2, \pi, 3 \pi / 2\}$ (displayed in blue), while "Shift 2 " is $\{0, \pi / 8,13 \pi / 8,15 \pi / 8\}$ (displayed in red). $\sqrt{2} \approx 1.4$; this can be compared with the uneven shift mentioned in the previous paragraph, where the condition number of $\mathrm{M}_{0}$ is increased to 13.2. Figure 2 shows the corresponding reconstruction results. In this simulation experiment, we synthesize the background intensity at value 100 , fringe contrast at value 50 , and ground truth phases from $-\pi / 2$ to $\pi / 2$, under independent identically distributed zeromean Gaussian noise with a standard deviation of 15. As predicted by the condition numbers, the even shift outperforms the uneven case, and the standard deviations of the error are $0.19 \mathrm{rad}$ and $0.95 \mathrm{rad}$, respectively.

- Equations (15) and (16) also help to shed light on the role of $F(x, \bar{y})$. In the low-SNR region, $F(x, y)$ is small, and therefore small errors or fluctuations in its reconstruction magnify the error of the reconstructed phase. This situation becomes worse after optical blurring because the fringe contrast of the sinusoidal signal is reduced. To show this, we synthesize the intensities with the fringe contrast value as above and compare the reconstruction results before and after blurring with the ground truth where the zero-mean Gaussian noise has a standard deviation of 10 . Figure 3 shows one of the experimental results in which the fringe contrast becomes $40 \%$ of the original. The standard deviation of the error after blurring is increased from 0.14 to $0.38 \mathrm{rad}$.

- An optimization framework allows us to incorporate prior knowledge, which can help to increase the accuracy of the reconstruction. In many applications, if the materials on the surface are locally homogeneous, the fringe contrast $F(x, y)$ is locally smooth. We can introduce a regularization on $F(x, y)$ when formulating the phase reconstruction problem. Accordingly, Eq. ( $\underline{8})$ is modified to

$$
E(x, y)=\sum_{k=1}^{n} E_{k}^{2}(x, y)+\lambda_{1} R_{1}(x, y)+\lambda_{2} R_{2}(x, y),
$$

where we use two regularization terms based on the local smoothness assumption on $F(x, y)$ along $x$ and $y$

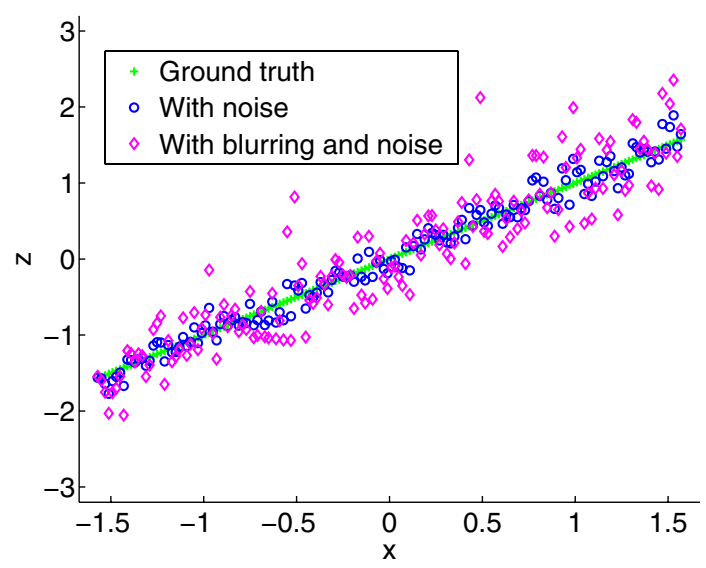

Fig. 3. (Color online) Reconstruction results measured in radians from the same PSA with and without blurring effect (displayed in magenta and blue, respectively). 
directions. $R_{1}(x, y)$ and $R_{2}(x, y)$ are given by

$$
\begin{aligned}
& R_{1}(x, y)=[F(x+1, y)-F(x, y)]^{2}, \\
& R_{2}(x, y)=[F(x, y+1)-F(x, y)]^{2},
\end{aligned}
$$

and $\lambda_{1}$ and $\lambda_{2}$ control the extent of such regularizations. Allowing two regularization terms caters for an anisotropic control of the smoothing in the $x$ and $y$ directions, which is useful when the imaging system has different magnifications along these directions.

- It is very important to note that, with $R_{1}(x, y)$ and $R_{2}(x, y)$, we can no longer minimize Eq. (11) point by point. Instead, we minimize the total energy, i.e.,

$$
\begin{aligned}
E_{T} & =\sum_{x, y} E(x, y) \\
& =\sum_{x, y, k} E_{k}^{2}(x, y)+\lambda_{1} \sum_{x, y} R_{1}(x, y)+\lambda_{2} \sum_{x, y} R_{2}(x, y) .
\end{aligned}
$$

We call this the regularized PSA (R-PSA).

\section{E. Solving the R-PSA}

We decompose the nonlinear optimization problem in Eq. (20) into three subproblems: initial estimation, regularization, and phase recovery. For each subproblem, we show how to solve it efficiently and ensure a unique global optimal solution.

1. Estimating the Initial Values for $B(x, y)$, $F(x, y)$ and $\phi(x, y)$. We approximate the regularization terms such that
With these quantities, the solution $\mathbf{v}$ is given by

$$
\mathbf{v}=\left(\mathrm{M}^{T} \mathrm{M}+\lambda_{1} \mathrm{D}_{x}{ }^{T} \mathrm{D}_{x}+\lambda_{2} \mathrm{D}_{y}{ }^{T} \mathrm{D}_{y}\right)^{-1} \mathrm{M}^{T} \mathbf{d} .
$$

In reality, to avoid handling large matrices, $\mathbf{v}$ is often solved using iterative schemes such as the conjugate gradient method [20].

The solution $\mathbf{v}$ provides us the initial estimations for $B(x, y), F(x, y)$, and $\phi(x, y)$, where the last term is obtained from $\arctan \left\{F_{s}(x, y) / F_{c}(x, y)\right\}$. However, due to noise, intensity fluctuation, or blurring, the reconstructed $F(x, y)$, especially in the low-SNR region, is inaccurate and unstable. We fix it in the next step.

2. Regularization on $F(x, y)$. We use the estimated values for $B(x, y)$ and $\phi(x, y)$ from the previous step and leave $F(x, y)$ as the unknown to be found. Referring back to Eq. (20), the expression is now quadratic in $F(x, y)$, and it can be solved efficiently. In a way very similar to the previous step, we can express the solution in an analytical form, but in reality we also solve by iterative schemes.

3. Phase recovery. Using $B(x, y)$ and the regularized $F(x, y)$ as the prior knowledge, we recover the phase $\phi(x, y)$ by solving the constrained optimization problem:

$\operatorname{minimize} \sum_{x, y, k}\left(I_{k}(x, y)-\left\{B(x, y)+F(x, y) \cos \left[\phi(x, y)+s_{k}\right]\right\}\right)^{2}$ suchthat

$$
\cos ^{2} \phi(x, y)+\sin ^{2} \phi(x, y)=1 .
$$

In this subproblem, we can solve $\cos \phi(x, y)$ and $\sin \phi(x, y)$ by a standard least-square optimization with a quadratic constraint [21].

$$
\begin{aligned}
& R_{1}(x, y) \approx\left[F_{c}(x+1, y)-F_{c}(x, y)\right]^{2}+\left[F_{s}(x+1, y)-F_{s}(x, y)\right]^{2} \\
& R_{2}(x, y) \approx\left[F_{c}(x, y+1)-F_{c}(x, y)\right]^{2}+\left[F_{s}(x, y+1)-F_{s}(x, y)\right]^{2}
\end{aligned}
$$

When $\phi(x+1, y)=\phi(x, y)$ and $\phi(x, y+1)=\phi(x, y)$, the above expressions can be simplified to Eqs. (18) and (19). For locally smooth surfaces with homogeneous reflectivity, these approximations are suitable for an initial estimation. Consequently, Eq. (20) is quadratic in $F_{c}(x, y)$ and $F_{s}(x, y)$, and we can solve for them efficiently.

In fact, there is an analytical solution. Let $\mathrm{M}$ be a block-diagonal matrix where the diagonal elements are $\mathrm{M}_{0}$, let $\mathbf{v}$ be a vector stacking $\mathbf{v}_{0}$ together by raster-scanning different $(x, y)$, and let $\mathbf{d}$ be formed from $\mathbf{d}_{0}$ is a similar fashion. The first term in Eq. (20) is then $\|\mathbf{M v}-\mathbf{d}\|_{2}^{2}$. Moreover, we can form difference matrices $\mathrm{D}_{x}$ and $\mathrm{D}_{y}$ so that

$$
\left\|\mathrm{D}_{x} \mathbf{v}\right\|_{2}^{2}=\sum_{x, y} R_{1}(x, y) \quad \text { and } \quad\left\|\mathrm{D}_{y} \mathbf{v}\right\|_{2}^{2}=\sum_{x, y} R_{2}(x, y) .
$$

In our formulation, we assume that $\lambda_{1}$ and $\lambda_{2}$ are positive constants during the initial estimation but will consider them as functions of $(x, y)$ and update them while regularizing $F(x, y)$. In fact, in our implementation we introduce two other constants, $C_{1}$ and $C_{2}$. The former is a measure of immunity to noise, which we set to 50 to avoid spurious discontinuities on $F(x, y)$ [22]. For $C_{2}$, we set it to be 250 , so that the contrast sensitivity threshold is about 15 gray levels. They can also be set based on the domain knowledge of the particular applications and how much regularization is desirable. We then let

$$
\lambda_{1}(x, y)=\frac{C_{1}}{C_{2}+R_{1}(x, y)},
$$

such that $\lambda_{1}(x, y) R_{1}(x, y) \approx C_{1} R_{1}(x, y) / C_{2} \quad$ when $R_{1}(x, y)$ is small but approaches $C_{1}$ when $R_{1}(x, y)$ gets large. Similarly, we set 


$$
\lambda_{2}(x, y)=\frac{C_{1}}{C_{2}+R_{2}(x, y)} .
$$

Using the above, we can reduce the dominant effect of the regularization term on the energy function at those points where the variations of $F(x, y)$ are large because the regularization term becomes the constant $C_{1}$. On the other hand, the regularization term becomes a convex quadratic cost function $C_{1} R_{1}(x, y) / C_{2}$ [or $C_{1} R_{2}(x, y) / C_{2}$ when we consider $\lambda_{2}$ ] in the smooth region where the variations of $F(x, y)$ are close to zero.

A summary of the above implementation is shown in Algorithm 1.

\section{Algorithm 1}

Input: (1) multiple images $I_{k}(x, y)$ and phase shifts $s_{k}$, where $k=\{1, \ldots, n\} ;(2)$ parameters $C_{1}$ and $C_{2}$ for the regularization terms.

Initialize the parameters $\lambda_{1}$ and $\lambda_{2}$ :

$\lambda_{1}=\lambda_{2}=C_{1} / C_{2}$.

1. Use the approximation in Eq. (21) to formulate the cost function $E_{T}$ in Eq. (20) into a standard positive definite matrix form of the variables $B(x, y)$, $F_{s}(x, y)$, and $F_{c}(x, y)$. We then solve $B(x, y), F_{s}(x, y)$, and $F_{c}(x, y)$.

2. Solve $F(x, y)$ and $\phi(x, y): \quad F(x, y)=$ $\sqrt{F_{s}(x, y)^{2}+F_{s}(x, y)^{2}}$ and $\phi(x, y)=\arctan \frac{F_{s}(x, y)}{F_{c}(x, y)}$.

3. Update the parameters $\lambda_{1}$ and $\lambda_{2}$ at the point $(x, y): \lambda_{1}(x, y)=\frac{C_{1}}{C_{2}+R_{1}(x, y)}$ and $\lambda_{2}(x, y)=\frac{C_{1}}{C_{2}+R_{2}(x, y)}$.

4. Use the $B(x, y), \phi(x, y), \lambda_{1}(x, y)$, and $\lambda_{2}(x, y)$ obtained above to formulate the cost function $E_{T}$ in Eq. (20) into a standard positive definite matrix form of the variables $F(x, y)$. Then refine $F(x, y)$ by minimizing this cost function.

5. Use $B(x, y)$ and the updated $F(x, y)$ to refine the $\phi(x, y)$ by solving a constrained optimization problem in Eq. (24).

6. Solve $\phi_{r}(x, y)$ on a reference plane in a similar fashion as in steps 1 to 5 . Use $\phi(x, y)$ and $\phi_{r}(x, y)$ to recover the surface $h(x, y)$ from Eqs. (2) and (1) . Output: the surface profile $h(x, y)$.

\section{Experimental Results}

\section{A. Accuracy for Nonuniform Shifts}

Our first experiment tests the improvement on accuracy of our proposed algorithm. Assuming a land grid

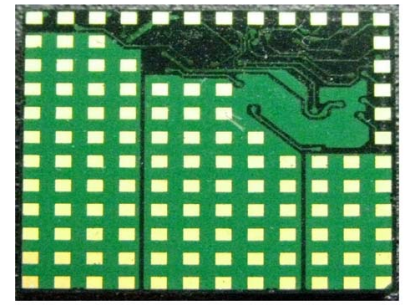

(a) The image of a LGA sample

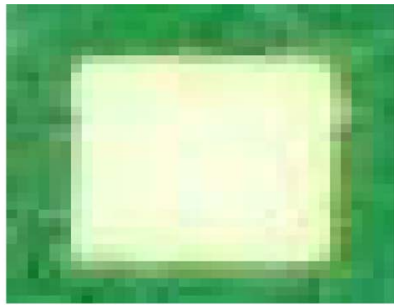

(b) A golden pad in LGA
Fig. 4. (Color online) Typical semiconductor sample called an LGA. (a) Image of an LGA sample; (b) golden pad in LGA.

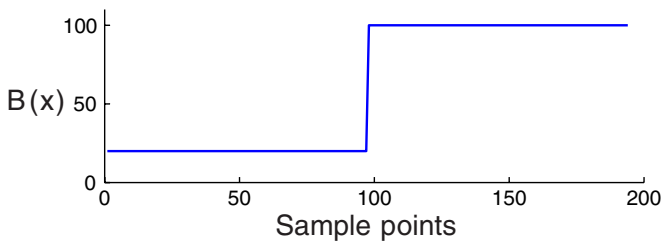

(a) The synthetic background intensity along the profile.

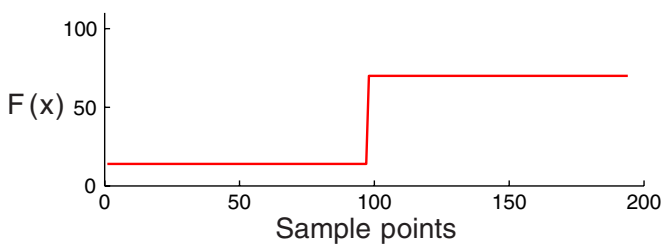

(b) The synthetic fringe contrast along the profile.

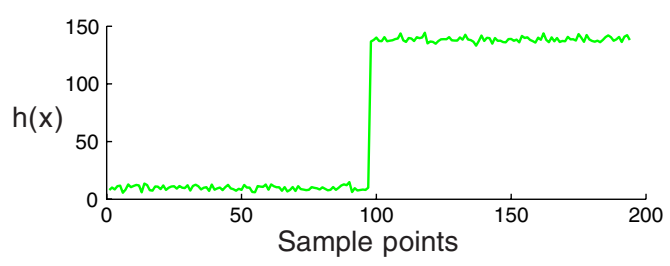

(c) A cross-section from the substrate to the golden pad.

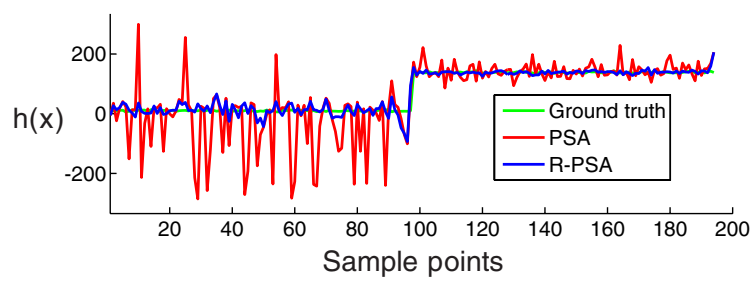

(d) Comparison of the results from PSA and R-PSA with four images.

Fig. 5. (Color online) Simulation experiments for comparing the improvement on accuracy between the PSA and the R-PSA. The reconstruction results are measured in micrometers. (a) Synthetic background intensity along the profile. (b) Synthetic fringe contrast along the profile. (c) Cross section from the substrate to the golden pad. (d) Comparison of the results from PSA and RPSA with four images.

array (LGA) package shown in Fig. $\underline{4}$, we synthesize the raw intensity images under typical conditions and compare the reconstruction results from PSAs and R-PSAs. Since most semiconductor packages are

Table 1. Comparison of the Standard Deviation of the Reconstruction Error

\begin{tabular}{lrrrr}
\hline & \multicolumn{4}{c}{ Noise Level } \\
\cline { 2 - 5 } & $\sigma=5$ & $\sigma=10$ & $\sigma=15$ & $\sigma=20$ \\
\hline PSA3 & 13.36 & 27.78 & 47.28 & 71.21 \\
PSA4 & 10.92 & 22.33 & 33.16 & 45.28 \\
PSA5 & 9.96 & 20.16 & 30.16 & 42.29 \\
R-PSA3 & 7.57 & 9.78 & 12.97 & 16.33 \\
R-PSA4 & 6.10 & 8.67 & 11.65 & 14.92 \\
R-PSA5 & 5.54 & 8.37 & 11.69 & 15.20 \\
\hline
\end{tabular}

Units in micrometers. 


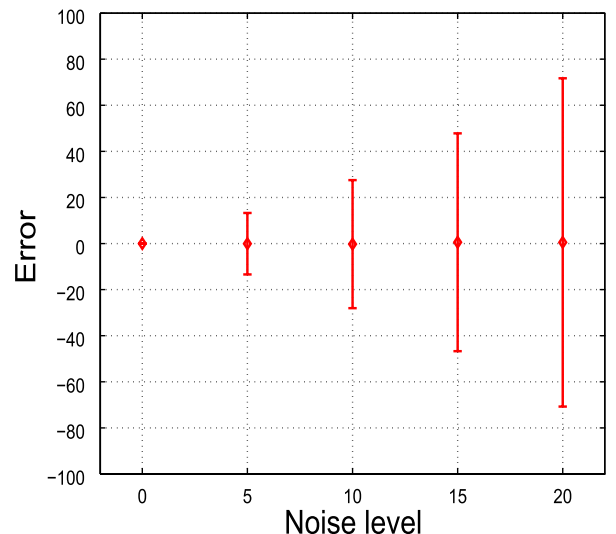

(a) Result from PSA3

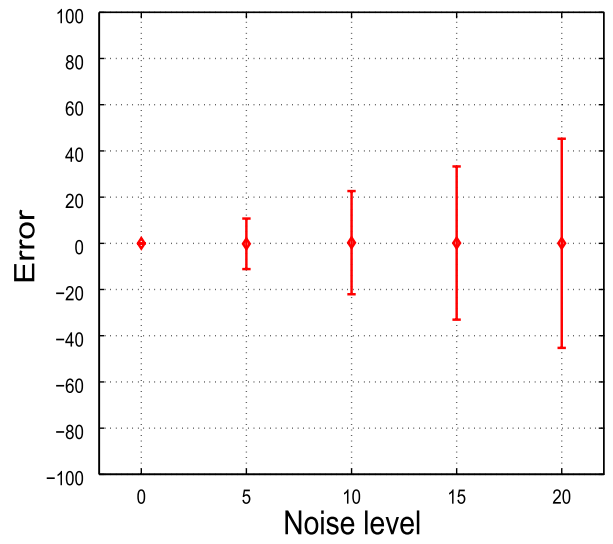

(c) Result from PSA4

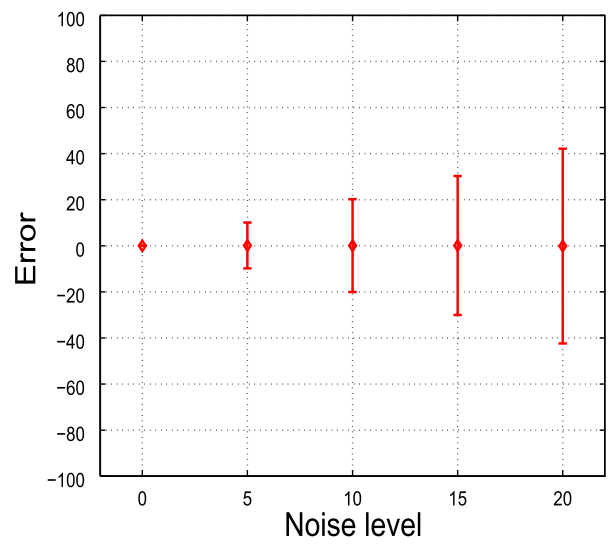

(e) Result from PSA5

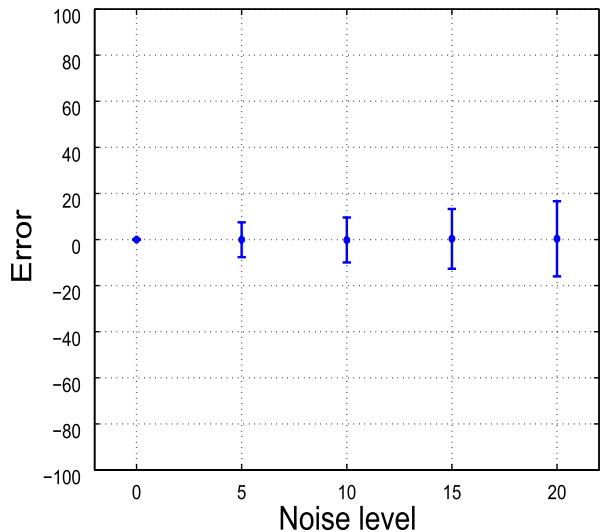

(b) Result from R-PSA3

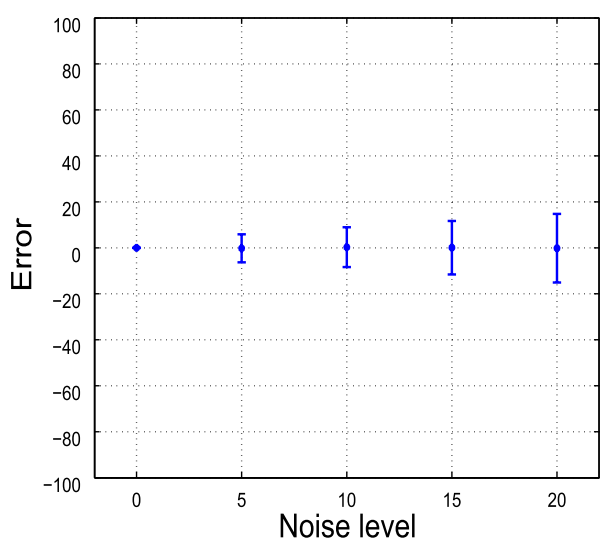

(d) Result from R-PSA4

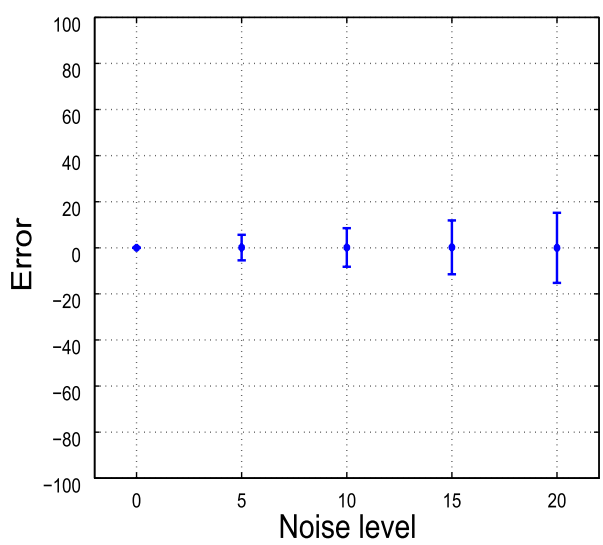

(f) Result from R-PSA5

Fig. 6. (Color online) Error ranges at different noise levels for PSA and R-PSA (units in micrometers). Results from (a) PSA3, (b) R-PSA3, (c) PSA4, (d) R-PSA4, (e) PSA5, (f) R-PSA5.

composed of a nonmetal substrate part and a metal part (the golden pad) and the reflectivities are locally homogeneous in the respective regions, we use step functions to represent the transition in both the background intensity and the fringe contrast. For the ground truth height profile, we use the values obtained by measuring a real LGA package in the substrate and the golden pad. We assume nonuniform phase shifts with $s_{1}=0, s_{2}=\pi / 8, s_{3}=13 \pi / 8$, and $s_{4}=15 \pi / 8$ as mentioned in Subsection 3.D, where the improvement using an R-PSA should be more pronounced. Accordingly, we synthesize four images based on the above, then blur them using a Gaussian filter with a unit standard deviation before adding some zero-mean Gaussian noise. Finally, we recover the height profile using a traditional PSA and the proposed R-PSA.

Figure 5 shows one set of the simulation results. In (a) and (b) we show the synthetic background intensity and fringe contrast; in (c) we have the measured 


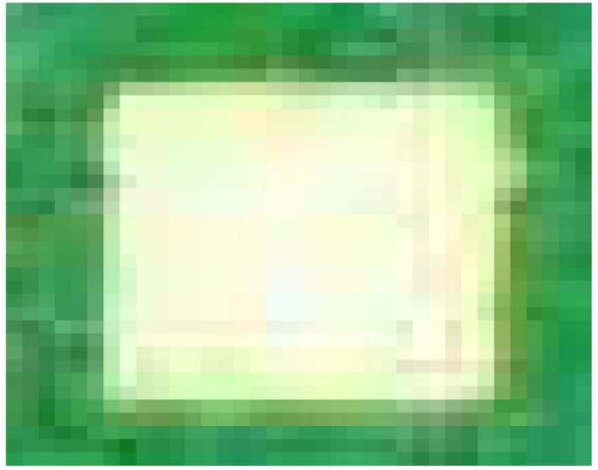

(a) The image of a golden pad

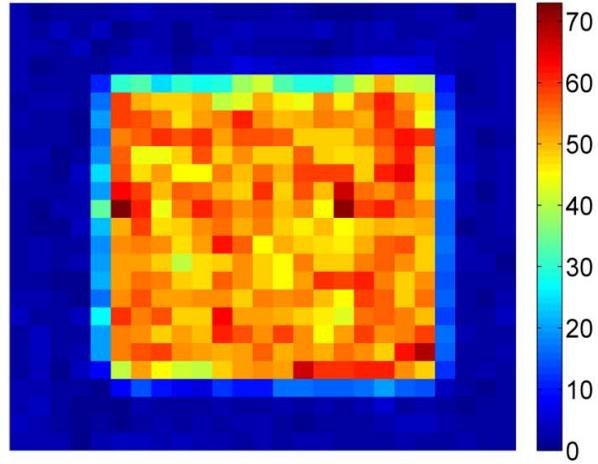

(c) The fringe contrast image

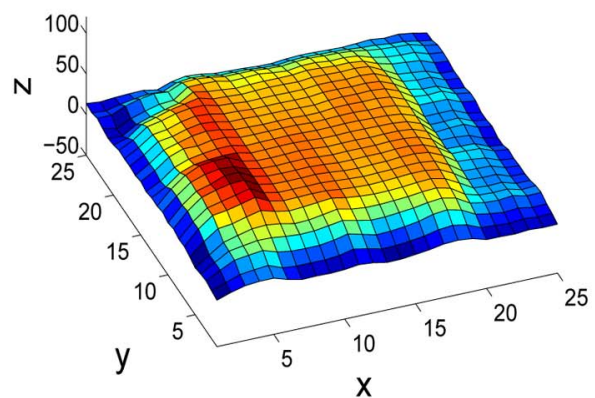

(e) The profile after smoothing

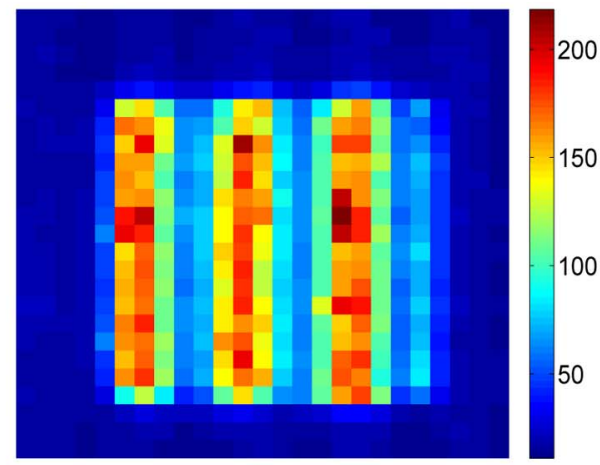

(b) The projected fringe image

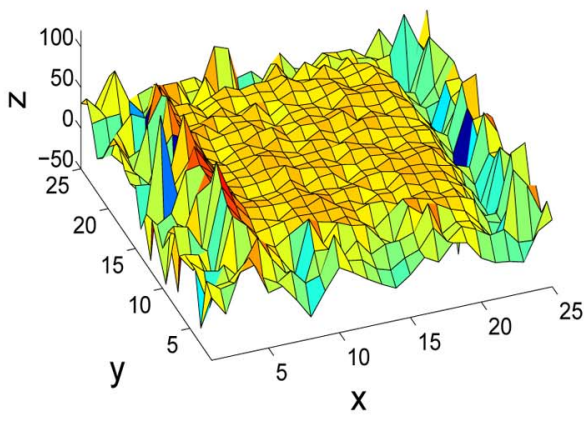

(d) The profile from PSA3

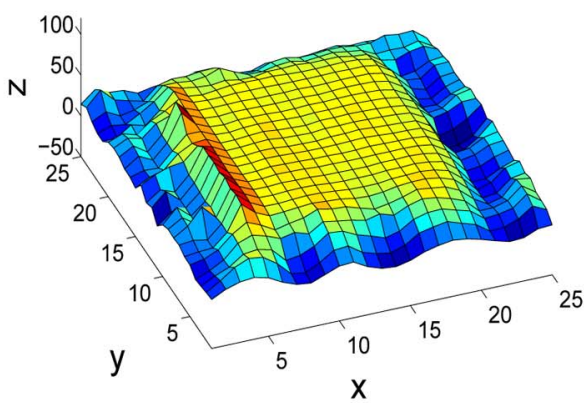

(f) The profile from R-PSA3

Fig. 7. (Color online) Surface reconstruction of a golden pad in an LGA sample. (a) Image of a golden pad. (b) Projected fringe image. (c) Fringe contrast image. (d) Profile from PSA3. (e) Profile after smoothing. (f) Profile from R-PSA3.

ground truth height profile. The latter is duplicated in (d), while we overlay the reconstruction results of the PSA and the R-PSA. It is evident that the R-PSA matches more closely with the ground truth than the PSA, not only in the high-SNR region (the golden pad) but also in the low-SNR region (the substrate). Numerically, the standard deviations of the errors have been reduced from 26.2 to $10.4 \mu \mathrm{m}$ in the former, and from 111.8 to $26.0 \mu \mathrm{m}$ in the latter.

\section{B. Robustness with Noise Levels}

Next we investigate the sensitivity of the general multiframe PSAs to additive noise at different levels. Here we assume uniform phase-shift values, i.e., $s_{k}=$ $(k-1) \pi / 2$ (for $k=1, \ldots, n$ ), where $n$ is the number of images. We experiment with $n=3, n=4$, and $n=5$, labeling the results from the PSAs as PSA3, PSA4, and PSA5, respectively; likewise, the reconstruction results from the R-PSAs are R-PSA3, R-PSA4, and RPSA5, respectively. We synthesize a randomly tilted plane and use this plane as the ground truth for comparison. First, we generate two random numbers within $[0,10]$ and $[0,360)$, respectively, as the inclination and azimuth angles in the spherical coordinate system for synthesizing the normal vector of this plane. We then retain only the center $20 \times 20$ pixels around the origin as the reconstruction region. Finally, we normalize the height profile within this region from -250 to $250 \mu \mathrm{m}$ to form the ground truth height. Meanwhile, the fringe contrast value $F(x, y)$ is a 


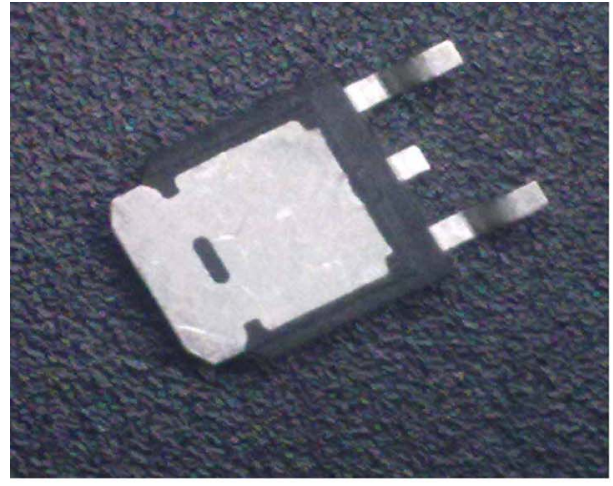

(a) The image of a DPAK sample

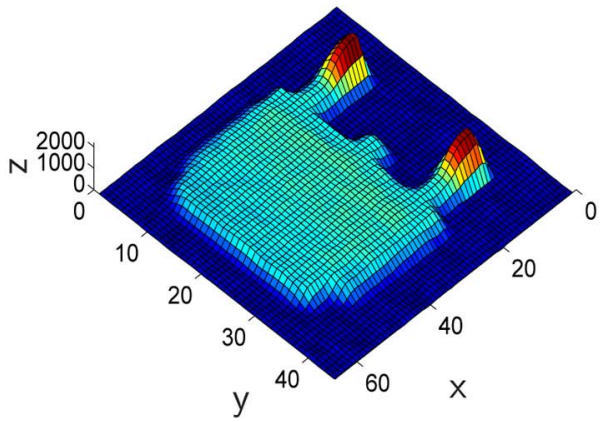

(c) The profile after smoothing

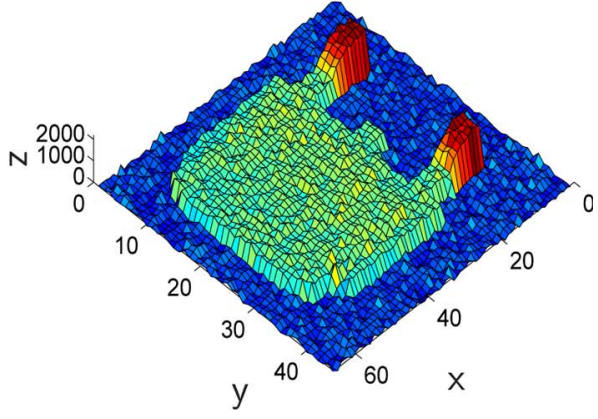

(b) The profile from PSA4

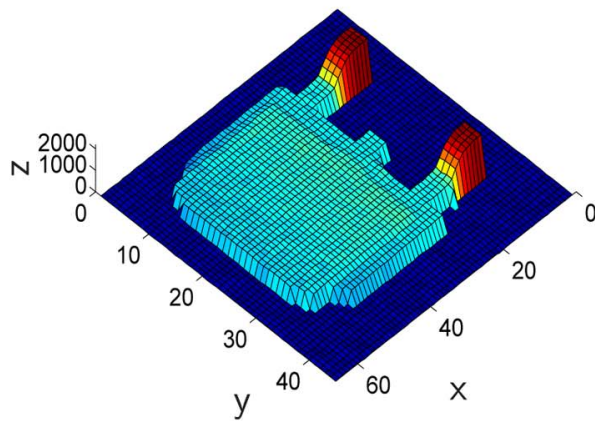

(d) The profile from R-PSA4

The 1D profile

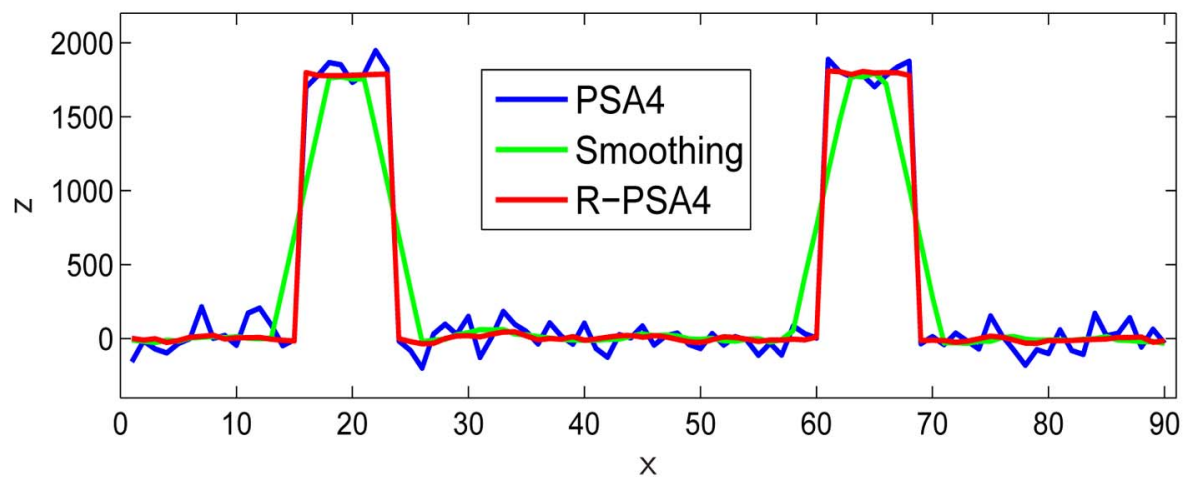

(e) Comparison of the cross-section on the column at $x=13$.

Fig. 8. (Color online) Surface reconstruction of a DPAK sample. (a) Image of a DPAK sample. (b) Profile from PSA4. (c) Profile after smoothing. (d) Profile from R-PSA4. (e) Comparison of the cross section on the column at $x=13$.

constant (80 out of 256 levels) with a normally distributed additive noise (with standard deviation $\sigma=5$ ), and the background intensity value $B(x, y)$ is set similarly (at 100 with $\sigma=5$ ). We then generate the images according to the model described in Section 2 . The noise level ranges from a standard deviation of $\sigma=5$ to $\sigma=20$, and for each case we test the surface reconstruction 100 times for different height profiles.

The results of these tests are summarized in Fig. $\underline{6}$ and in Table 1 . The former gives a graphical view of the error range ( 1 standard deviation wide in each direction) for different methods under various noise levels, and the thick dots represent the averages of the reconstruction errors. The symmetry of these figures suggests that there is no bias in both PSAs and the R-PSAs. More importantly, by looking at the standard deviation of the resulting errors, we can observe that they increase substantially with respect to the noise level for PSAs, and the increase is more drastic with fewer images. While a similar trend is also observed in the R-PSA, the error range stays small even with a higher noise level, and reducing the number of captured images from five to three does not have much effect in reducing the robustness especially at a high noise level. At a low noise level, 
more images still make better R-PSA results, as seen in Table $\underline{1}$.

\section{Application Using a Real Semiconductor Package}

Figure 7 shows an experimental result involving a golden pad in an LGA sample. In the green substrate region, which has a low SNR, the fringe pattern is weak as shown in (b), so it is challenging to obtain an accurate height profile. However, since the materials are homogenous, the fringe contrasts are locally smooth, as shown in (c). If we do not make use of this assumption, using a traditional PSA3 reconstruction, we obtain the noisy profile in (d). We can apply a smoothing filter to suppress the noise, as shown in (e); however, the abrupt height change from the substrate to the golden pad becomes more gradual, and the outliers on the left boundary of the golden pad cause deformation on the profile.

On the other hand, incorporating the smoothness regularization in both the substrate and the golden pad regions, we can achieve better profile results. This is shown in Fig. 7(f). Not only do we reduce the noise, but we also keep the sharp transition in height from the substrate to the golden pad. The resulting profile is more realistic and is insensitive to outliers.

Figure (8) shows another reconstruction result, this time with a typical discrete package (DPAK) sample. The result from the PSA is noisy, as shown in (b). Again, if we apply an averaging filter directly on the result, noise can be reduced at the expense of deformation in the shape of the metal lead and the boundary of the DPAK, shown in (c). With regularization, we simultaneously suppress the noise and keep sharp transitions, as depicted in (d).

\section{Conclusion}

In this paper, we investigate the PSA from an optimization point of view and incorporate a smoothness constraint as regularization. Thus, we not only use the interframe correlation but also explore the spatial correlation on the surface. We call this the RPSA and show experimentally that it outperforms the PSA in terms of accuracy and robustness.

This work is supported in part by ASM Assembly Automation Limited and by the University Research Committee of the University of Hong Kong under project 10208648 .

\section{References}

1. A. N. Ng, E. Y. Lam, R. Chung, K. S. Fung, and W. Leung, "Reference-free machine vision inspection of semiconductor die images,” Int. J. Image Graphics 9, 133-152 (2009).
2. G. Zeng, Y. Matsushita, L. Quan, and H.-Y. Shum, "Interactive shape from shading," in IEEE Computer Society Conference on Computer Vision and Pattern Recognition (IEEE, 2005), pp. 343-350.

3. M. Ishihara and H. Sasaki, "High-speed 3D shape measurement using a nonscanning multiple-beam confocal imaging system," Proc. SPIE 3478, 68-75 (1998).

4. D. Scharstein and R. Szeliski, "A taxonomy and evaluation of dense two-frame stereo correspondence algorithms," Int. J. Comput. Vis. 47, 7-42 (2002).

5. D. Caspi, N. Kiryati, and J. Shamir, "Range imaging with adaptive color structured light," IEEE Trans. Pattern Anal. Mach. Intell. 20, 470-480 (1998).

6. X. Su and W. Chen, "Fourier transform profilometry: a review," Opt. Lasers Eng. 35, 263-284 (2001).

7. V. Srinivasan, H. Liu, and M. Halioua, "Automated phasemeasuring profilometry: a phase mapping approach," Appl. Opt. 24, 185-188 (1985).

8. J. Cheng, R. Chung, E. Y. Lam, and K. S. Fung, "Bit-pairing codification for binary pattern projection system," in International Conference on Pattern Recognition (IEEE, 2006), Vol. 2, pp. 263-266.

9. J. Cheng, R. Chung, E. Y. Lam, K. S. Fung, and Y. Xu, "Optimization of bit-pairing codification with learning for 3D reconstruction," Int. J. Image Graphics 7, 445-462 (2007).

10. J. Salvi, J. Pagés, and J. Batlle, "Pattern codification strategies in structured light systems," Pattern Recogn. 37, 827-849 (2004).

11. M. Dong, R. Chung, E. Y. Lam, and K. S. Fung, "Height inspection of wafer bumps without explicit 3D reconstruction," IEEE Trans. Electron. Packag. Manuf. 33, 112-121 (2010).

12. J. Cheng, R. Chung, E. Y. Lam, K. S. Fung, F. Wang, and W. Leung, "Structure-light based sensing using a single fixed fringe grating: fringe boundary detection and 3D reconstruction," IEEE Trans. Electron. Packag. Manuf. 31, 19-31 (2008).

13. V. Srinivasan, H. Liu, and M. Halioua, "Automated phasemeasuring profilometry of 3-D diffuse objects," Appl. Opt. 23, 3105-3108 (1984).

14. C. Ai and J. C. Wyant, "Effect of spurious reflection on phase shift interferometry," Appl. Opt. 27, 3039-3045 (1988).

15. C. P. Brophy, "Effect of intensity error correlation on the computed phase of phase-shifting interferometry," J. Opt. Soc. Am. A 7, 537-541 (1990).

16. P. J. de Groot, "Vibration in phase-shifting interferometry," J. Opt. Soc. Am. A 12, 354-365 (1995).

17. P. Hariharan, B. F. Oreb, and T. Eiju, "Digital phaseshifting interferometry: a simple error-compensating phase calculation algorithm," Appl. Opt. 26, 2504-2506 (1987).

18. Y. Shu, R. Chung, Z. Tan, J. Cheng, E. Y. Lam, K. S. Fung, and F. Wang, "Projection optics design for tilted projection of fringe pattern," Opt. Eng. 47, 053002 (2008).

19. S. Boyd and L. Vandenberghe, Convex Optimization (Cambridge University, 2004).

20. C. C. Paige and M. A. Saunders, "LSQR: an algorithm for sparse linear equations and sparse least squares," ACM Trans. Math. Softw. 8, 43-71 (1982).

21. A. Björck, Numerical Methods for Least Squares Problems (Society for Industrial and Applied Mathematics, 1996).

22. A. Blake and A. Zisserman, Visual Reconstruction (MIT, 1987). 